\title{
The application of fixed hydrophobic patterns for confinement of aqueous solutions in proteomic microarrays
}

Y. Li, E. O. McKenna, W. Parkes, A. R. Pitt, and A. J. Walton

Citation: Appl. Phys. Lett. 99, 073703 (2011); doi: 10.1063/1.3626037

View online: https://doi.org/10.1063/1.3626037

View Table of Contents: http://aip.scitation.org/toc/apl/99/7

Published by the American Institute of Physics

\section{Articles you may be interested in}

Formation of superhydrophobic/superhydrophilic patterns by combination of nanostructure-imprinted perfluoropolymer and nanostructured silicon oxide for biological droplet generation

Applied Physics Letters 98, 123706 (2011); 10.1063/1.3570627

Electrowetting-based actuation of liquid droplets for microfluidic applications

Applied Physics Letters 77, 1725 (2000); 10.1063/1.1308534

Electro-wetting displays

Applied Physics Letters 38, 207 (1981); 10.1063/1.92322

Variable-focus liquid lens for miniature cameras

Applied Physics Letters 85, 1128 (2004); 10.1063/1.1779954

\section{COMSOL} CONFERENCE 2018 BOSTON 


\title{
The application of fixed hydrophobic patterns for confinement of aqueous solutions in proteomic microarrays
}

\author{
Y. Li, ${ }^{1, a)}$ E. O. McKenna, ${ }^{2}$ W. Parkes, ${ }^{1}$ A. R. Pitt, ${ }^{2,3}$ and A. J. Walton ${ }^{1}$ \\ ${ }^{1}$ Scottish Microelectronic Centre, IMNS, School of Engineering, University of Edinburgh, Edinburgh \\ EH9 3JF, United Kingdom \\ ${ }^{2}$ Institute of Molecular, Cell and Systems Biology, College of Medical, Veterinary and Life Sciences, University \\ of Glasgow, Glasgow G12 8QQ, United Kingdom \\ ${ }^{3}$ School of Life and Health Sciences, Aston University, Aston Triangle, Birmingham B4 7ET, United Kingdom
}

(Received 8 April 2011; accepted 27 July 2011; published online 18 August 2011)

A protein microarray hybridisation system has been implemented by employing patterned hydrophobic thin films on hydrophilic substrates as a means of confinement for aqueous samples. This approach has the ability to handle, and keep separate, small sample volumes of just a few microlitres. In addition, the system is more straightforward to use than the existing multi-well gasket solution. The paper describes the fabrication method and the system is demonstrated for a model protein microarray assay. (C) 2011 American Institute of Physics. [doi:10.1063/1.3626037]

There is currently a broad interest in analytical formats involving micro-features spotted onto planar surfaces for biological applications, such as in the study of proteins, DNA, lipids, and cells. ${ }^{1-5}$ Such planar systems on microscope slides are often termed "biochips" or "microarrays" and they require a reliable means of applying and corralling microlitre scale liquid samples. Existing systems designed for use with arrays made on microscope slides typically use a frame of chambers or wells and a gasket to keep liquid samples separated, preventing mixing of hybridisation liquid between sections of the protein microarray slides, as schematically illustrated in Fig. 1. Such wells usually require the use of several tens of microlitres of sample fluid and are prone to problems related to the nature of the gasket structure. These include cross-contamination between adjacent liquid pools caused by gasket leaks, non-uniformity caused by air bubbles, evaporation, and issues associated with the liquid meniscus present with small sample volumes.

Fig. 2 presents an improved approach for keeping small volumes of liquid separated using wall-less reaction chambers, which simply employs surface tension to confine the liquid. Hence, the gasket shown in Fig. 1 is replaced with a patterned hydrophobic surface that separates the liquid hybridisation samples (Fig. 2(a)). The microarray slide is placed on top of the array of liquid droplets, the separation of the two facing surfaces being determined using gap-defining spacers $(\sim 100$ $\mu \mathrm{m}$ thick). The parallel plate structure defined by these spacers ensures a pre-defined and uniform thickness of liquid across the protein array, with the shape of the contact areas of the sample liquid on the protein microarray slide being set by the hydrophilic pattern on the substrate.

Fig. 3 compares the differences in microarray spot signal uniformity obtained from gasket confinement and the wall-less format. The two slides shown were spotted with BSA (bovine serum albumin) and probed using an anti-BSA antibody. The slides were processed in parallel with reagents from the same batch; hence, intensity variations observed

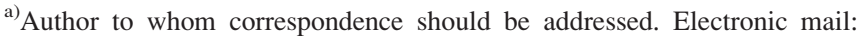
Y.Li@ed.ac.uk.
}

can mainly be attributed to the method of sample application. Fig. 3(a) reveals some frequently observed non-uniformities of the average spot fluorescent signal of each square arrays when using a gasket-based format. These non-uniformities greatly affect the precision and accuracy of the microarray analysis. Importantly, effects of this nature usually only become apparent at the end of the experiment. Even worse, in cases when a range of concentrations or biological compositions are used in different wells, these issues may escape undetected and lead to incorrect conclusions. In comparison, Fig. 3(b) shows the improved uniformity across the slide with the wall-less containment system, resulting in a more uniform spot fluorescent signal (the inter-block coefficient of variation $(\mathrm{CV})$ is reduced from $29.8 \%$ to $8.6 \%$ ). Even when the gasket frame technology produces equally uniform results such as the two middle blocks in Fig. 3(a), the sporadic unpredictable nature of the effect introduces uncertainty in the result.

Patterned hydrophobic/hydrophilic surfaces have been employed in a number of microfluidic applications for liquid manipulation using surface tension directed capillary force. ${ }^{6-14}$ In this work, they have been used to divide liquid samples in a protein microarray application, which requires that each droplet in the array is kept separate, i.e., clear hydrophobic and hydrophilic zones to provide stable reliable

(a)

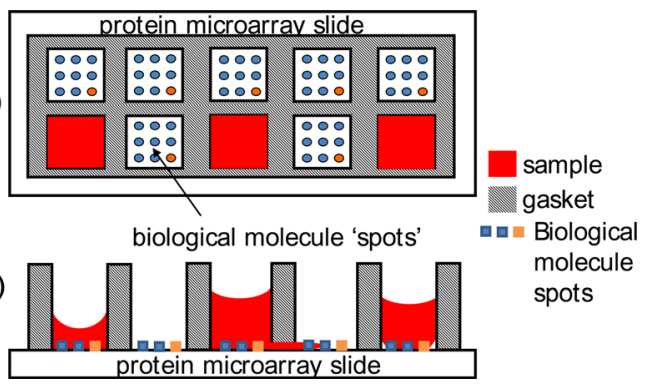

FIG. 1. (Color online) A schematic representation of a micro-array slide using conventional gasket-based confinement system showing of three aqueous liquid samples in separate wells interacting with a microarray slide. (a) Top view and (b) side view. 
(a)

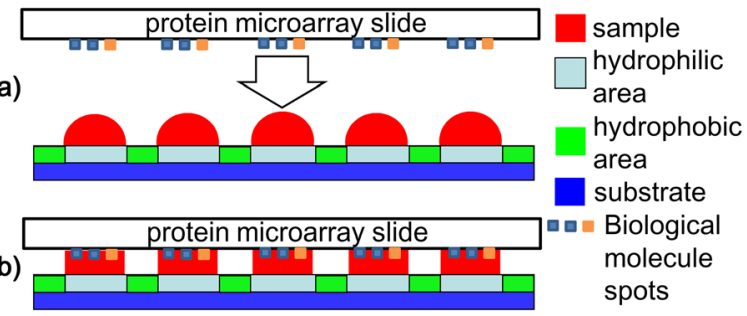

FIG. 2. (Color online) A schematic cross-section of (a) protein micro-array slide separated from the substrate with a hydrophilic surface patterned with a hydrophobic layer for liquid sample confinement and (b) hydrophobic patterned surface in a "sandwich" with the microarray slide.

"virtual" walls. In this study, Teflon ${ }^{\circledR}-\mathrm{AF}, \mathrm{CYTOP}^{\mathrm{TM}}$, and Parylene- $\mathrm{C}^{\mathrm{TM}}$ were selected as hydrophobic surface candidates that are biologically and chemically inert. ${ }^{15}$ Thermally grown $\mathrm{SiO}_{2}$ on $\mathrm{Si}$ provided the hydrophilic substrate.

Figs. 4(a)-4(c) shows the fabrication sequence used to create patterns on silicon with a high wettability contrast. A layer of $\mathrm{SiO}_{2}$, which provides the hydrophilic substrate, is initially thermally grown on silicon, followed by the deposition of a hydrophobic layer. An adhesion promoter is typically required before photoresist is coated onto hydrophobic surfaces such as Teflon-AF and CYTOP ${ }^{\circledR},{ }^{16}$ which can reduce the surface hydrophobicity. For example, the contact angles (a measure of surface hydrophobicity ${ }^{17}$ ) on Teflon$\operatorname{AF}\left(120^{\circ}\right)$ and $\operatorname{CYTOP}\left(114^{\circ}\right)$ were found to reduce to $104^{\circ}$ after surfactant Zonyl ${ }^{\circledR}$ FSN treatment, while a worse degradation is observed with oxygen plasmas. However, high viscosity photoresist Shipley SPR ${ }^{\mathrm{TM}} 220-3$ and 220-7 proved to be compatible with both Parylene- $\mathrm{C}^{\circledR}$ and CYTOP without compromising hydrophobicity. Standard photolithography was employed to pattern the resist, which was then used as a mask while oxygen plasma was used to selectively remove the hydrophobic coating to expose hydrophilic areas of $\mathrm{SiO}_{2}$. The resulting surfaces were evaluated for suitability by observing the contact angles, which were $114^{\circ}$ for CYTOP and $89^{\circ}$ for Parylene-C. Fig. 4(d) shows the mask layout of a hydrophilic pattern on hydrophobic surface, and Fig. 4(e)

(a)

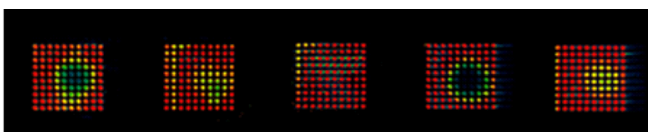

(b)

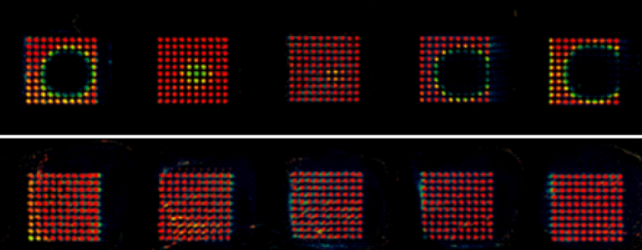

)

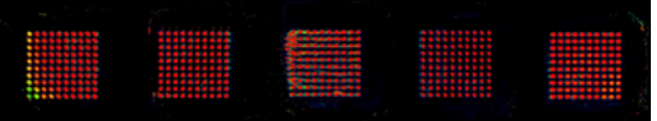

FIG. 3. (Color online) Fluorescent scans of two APTES microarray slides containing identical $10 \times 10$ spot array blocks $(500 \mu \mathrm{m}$ spot-to-spot BSAanti-BSA assay) processed using: (a) a gasket frame (70 $\mu$ l sample per well requires to be added), which shows a particularly poor example of the variation of the spot signal that can be observed with such a multi-well microarray and (b) hydrophobic patterned chip with square features ( $7 \mu$ sample per "well"), which illustrates improved uniformly.
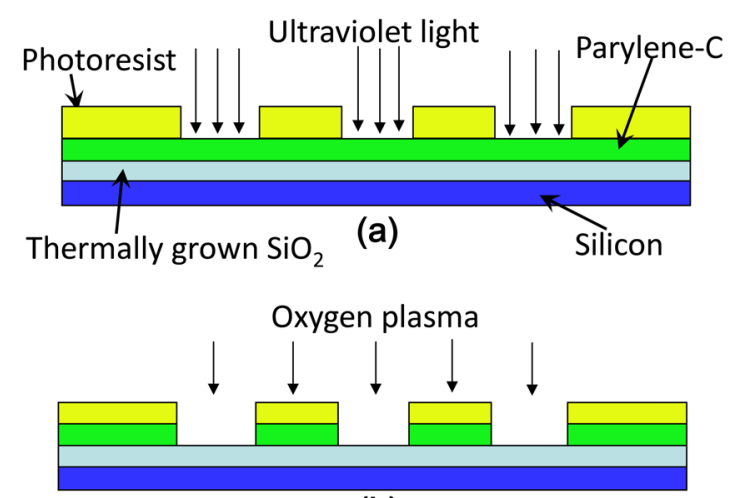

(b)

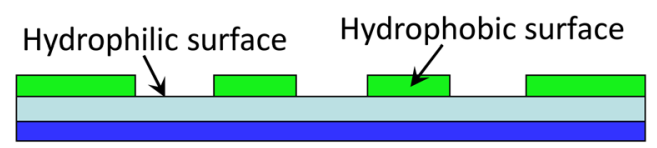

(c)

(d)
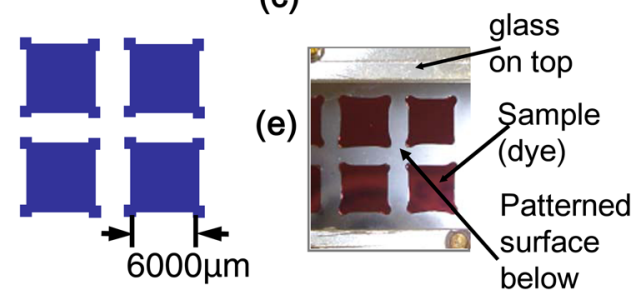

FIG. 4. (Color online) Cross-section of the processing steps used to fabricate the wall-less hybridisation chamber array on a silicon wafer: (a) photoresist exposure and development, (b) oxygen plasma etching of Parylene-C, (c) photoresist removal; and the top view of (d) hydrophilic mask patterns, (e) assembled device with hydrophilic areas filled with sample dye.

shows patterned hydrophilic areas filled with sample dye after system assembly with the cover-glass on top.

The model microarray assay used to test the concept used a reverse-phase type-BSA (Roche Diagnostics Ltd.) immobilised on the surface and probed with anti-BSA antibody. Inhouse prepared glass slides silanized with 3-(aminopropyl) triethoxysilane (APTES, Sigma) and also commercial thin nitrocellulose microarray slides $\left(\mathrm{PATH}^{\mathrm{TM}}\right.$ plus, GenTel Biosciences) were spotted using a Piezorray ${ }^{\mathrm{TM}}$ arrayer (Perkin Elmer) with volumes of $1 \mathrm{nl}$ of $0.2 \mathrm{mg} / \mathrm{ml} \mathrm{BSA}$ in phosphate buffer saline (PBS). These were on a $10 \times 10$ grid with a centre to centre spot spacing of $500 \mu \mathrm{m}$ and with a pitch of $9 \mathrm{~mm}$ between the array blocks. The arrays were blocked with $1 \% \beta$-lactoglobulin (Sigma) in PBS for $1 \mathrm{~h}$ in a slide mailer, and the slide edges dried on a paper towel and the slides air dried. Anti-BSA polyclonal whole rabbit serum (SigmaAldrich) at 1:1000 dilutions in PBS was then applied to the array for $1 \mathrm{~h} .7 \mu \mathrm{l}$ per $10 \times 10$ pad was used for the wall-less approach and compared with the conventional gasket frame (ArraySlide, Gel Company), which was filled with $70 \mu \mathrm{l}$. After the first binding reaction, the microarray/patterned surface assembly was flooded with PBST (PBS containing $0.1 \%$ Tween20). The modular nature then allowed the array slide to be lifted with tweezers, placed in a slide chamber and washed further with PBST. The patterned chip was also washed in a separate vessel at the same time. The gasket frames were emptied of probe solution, and the arrays washed in situ with PBST. All components were then briefly flushed with dry nitrogen, reassembled, and a solution of AlexaFluor647 
(a)

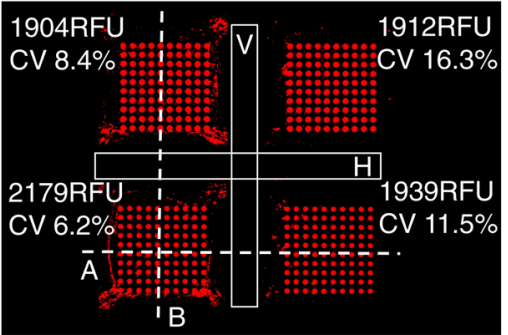

(c)

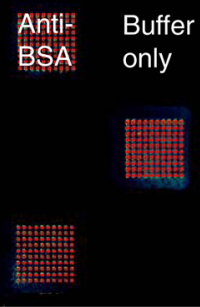

Average Background (region V) $=26 \mathrm{RFU}$ Average Background (region H) $=25$ RFU

(b)

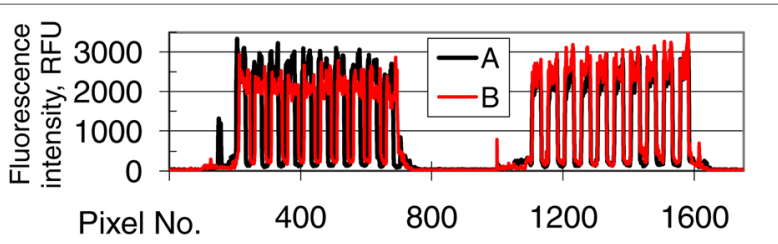

FIG. 5. (Color online) (a) A fluorescent image at resolution of $10 \mu \mathrm{m}$ per pixel of four microarray blocks of BSA, 100 spots each, $10 \times 10$ grid, $500 \mu \mathrm{m}$ spot-to-spot, APTES surface processed using $7 \mu \mathrm{l}$ of anti-BSA serum at 1:1000 per well, with block average spot signals (in relative fluorescence units, RFU) and CVs. (b) Fluorescence intensity profiles in the middle of the probed areas. (c) An example of an image of an array probed with the use of the patterned chip for cross-contamination.

labelled anti-rabbit antibody (Invitrogen) applied for $30 \mathrm{~min}$. Slides were then processed as before, dried, and scanned on a Perkin Elmer ScanArrayExpress. Fluorescence intensity profiles indicated that the good registration between the microarrays and the wall-less sample "wells" on the chip ensured the samples remained separated and confined during sample application and probing (Figs. 5(a) and 5(b)). Furthermore, when a similar experiment was carried out to assess crosscontamination, in which every other well was filled with the buffer without the anti-BSA antibody in a checkerboard pattern, no cross-contamination was observed for the wall-less assembly (Fig. 5(c)).

To conclude, we have demonstrated that the use of a hydrophobically patterned $\mathrm{SiO}_{2}$ surface provides a robust and simple to use platform for probing a multi-well microarray, producing either similar or higher quality results from 10 times smaller sample volumes than conventional gasket systems. This approach is based on surface patterning and offers design flexibility. It works well with a commonly used wettable microarray surfaces, and the fabrication method yields robust surfaces for exploiting capillary phenomena in microarray processing devices. The advantages are particularly apparent in biological microarray applications where sample volumes are limited and dilution of the probes is undesirable. This technology can also be applied to a wider range of applications, such as controlling nucleation, moisture trapping, etc. ${ }^{18-20}$ The ability to generate small features in the hydrophilic pattern perfectly complements arrays of very small lateral dimensions that are becoming available due to advances in microfabrication technology.

This research was supported by the BBSRC Interdisciplinary Research Collaboration in Proteomic Technologies: RASOR (BBC5115991). Authors declare competing commercial interest.

${ }^{1}$ P. F. Predki, Curr. Opin. Chem. Biol. 8, 8 (2004).

${ }^{2}$ M. F. Templin, D. Stoll, J. Bachmann, and T. O. Joos, Comb. Chem. High Throughput Screening 7, 223 (2004).

${ }^{3}$ P. Angenendt, Drug Discovery Today 10(7), 503 (2005).

${ }^{4}$ M. Schena, D. Shalon, R. W. Davis, and P. O. Brown, Science 270, 467 (1995).

${ }^{5}$ P. Cullen and S. Lorkowski, Expert Opin. Ther. Patents 12, 1 (2002).

${ }^{6}$ T. Kobayashi, K. Shimizu, Y. Kaizuma, and S. Konishi, Appl. Phys. Lett. 98, 123706 (2011).

${ }^{7}$ V. Kekkonen, A. Hakola, T. Kajava, E. Sahramo, J. Malm, M. Karppinen, and R. A. A. Ras, Appl. Phys. Lett. 97, 044102 (2010).

${ }^{8}$ D. Juncker, Doctor of Science thesis, Universite de Neuchatel, Zurich, 2002.

${ }^{9}$ B. Zhao, J. S. Moore, and D. J. Beebe, Science 291, 1023 (2001).

${ }^{10}$ A. A. Darhuber and S. M. Troian, Phys. Rev. E 64, 031603 (2001).

${ }^{11}$ S. Bouaidat, O. Hansen, H. Bruus, C. Berendsen, N. K. Bau-Madsen, P. Thomsen, A. Wolff, and J. Jonsmann, Lab Chip 5, 827 (2005).

${ }^{12}$ P. Lam, K. J. Wynne, and G. E. Wnek, Langmuir 18(3), 948 (2002).

${ }^{13}$ J. W. Suk and J. H. Cho, J. Micromech. Microeng. 17, N11 (2007).

${ }^{14}$ M. Watanabe, Sens. Actuators B 122, 141 (2007).

${ }^{15}$ C. P. Tan and H. G. Craighead, Materials 3, 1803 (2010).

${ }^{16}$ C. Cho, R. Wallace, and L. Files-Sesler, J. Electron. Mater. 23, 827 (1994).

${ }^{17}$ K. L. Mittal, Contact Angle, Wettability and Adhesion: Festschrift in Honor of Prof. Robert J. Good (VSP, Utrecht, the Netherlands, 1993).

${ }^{18}$ J. Aizenberg, A. J. Black, and G. M. Whitesides, Nature 398, 495 (1999).

${ }^{19}$ K. K. Varanasi, M. Hsu, N. Bhate, W. Yang, and T. Deng, Appl. Phys. Lett. 95, 094101 (2009).

${ }^{20}$ L. Zhai, M. C. Berg, F. C. Cebeci, Y. Kim, J. M. Milwid, M. F. Rubner, and R. E. Cohen, Nano Lett. 6, 1213 (2006). 